\title{
Accuracy and completeness of electronic medical records obtained from referring physicians in a Hamilton, Ontario, plastic surgery practice: A prospective feasibility study
}

\author{
Chris Joon Hong BHSc${ }^{1}$, Manraj Nirmal Kaur PT MSc ${ }^{2,3}$, Forough Farrokhyar PhD ${ }^{3,4}$, Achilleas Thoma MD MSc ${ }^{2,3,4}$
}

\begin{abstract}
CJ Hong, MN Kaur, F Farrokhyar, A Thoma. Accuracy and completeness of electronic medical records obtained from referring physicians in a Hamilton, Ontario, plastic surgery practice: A prospective feasibility study. Plast Surg 2015;23(1):48-50.
\end{abstract}

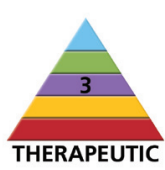

OBJECTIVE: To assess the feasibility of auditing electronic medical records (EMRs) in plastic surgery for future large-scale research studies. The secondary objective was to ascertain the accuracy and completeness of EMRs accompanying referral requests by physicians for plastic surgery consultation between July and December 2013.

METHODS: EMRs of 30 patients were reviewed and crosschecked independently by two reviewers and subsequently verified by a third reviewer using predefined criteria to determine whether they were accurate and/or complete. Descriptive analysis was performed to calculate the frequency of inaccuracies and incompleteness for each EMR information field. Information fields were compared to assess whether the frequency of inaccuracies and incompleteness varied.

RESULTS: Of the 270 information fields reviewed, four $(1.48 \%)$ were inaccurate and $66(24.4 \%)$ were incomplete. The most common field of inaccuracy was current medications, followed by medical history and medical allergies. The most common field of incompleteness was history of presenting illness followed by surgical history.

CONCLUSION: Despite their purported benefits, inaccuracies and incompleteness are a frequently occurring problem in EMRs. A large-scale study may be beneficial in determining the efficacy of EMRs in the future.

Key Words: Electronic health record; Electronic medical records; Feasibility study; History taking
L'exactitude et l'exhaustivité des dossiers médicaux électroniques des médecins orienteurs d'un cabinet de chirurgie plastique de Hamilton, en Ontario : une étude prospective de faisabilité
OBJECTIF : Évaluer la faisabilité de vérifier les dossiers médicaux électroniques (DMÉ) en chirurgie plastique en vue de futurs projets de recherche à grande échelle. L'objectif secondaire visait à établir l'exactitude et l'exhaustivité des DMÉ accompagnant les demandes d'aiguillage de médecins vers une consultation en chirurgie plastique entre juillet et décembre 2013.

MÉTHODOLOGIE : Deux réviseurs, suivis d'un troisième, ont examiné et contre-vérifié les DMÉ de 30 patients de manière indépendante au moyen de critères prédéfinis pour déterminer s'ils étaient exacts ou complets. Ils ont effectué une analyse descriptive pour calculer la fréquence d'inexactitudes et d'omissions dans chaque champ d'information des DMÉ. Ils ont comparé les champs d'information pour évaluer la variation de la fréquence d'inexactitudes et d'omissions.

RÉSULTATS : Sur les 270 champs d'information examinés, quatre $(1,48 \%)$ étaient inexacts et 66 (24,4\%), incomplets. Le champ grevé du plus d'inexactitudes était les médicaments actuels, suivi des antécédents médicaux et des allergies médicales. Le champ le plus souvent incomplet était l'histoire des symptômes initiaux, suivi des antécédents chirurgicaux. CONCLUSION : Malgré les prétendus avantages des DMÉ, les inexactitudes et les omissions sont fréquentes. Une étude à grande échelle pourrait contribuer à déterminer l'efficacité des DMÉ.
$\mathrm{T}$ here has been mounting recognition of the role of information technology systems in the provision of health care. Electronic medical records (EMRs) - an initiative of the 1970s - have measurably enhanced the quality of health care. They facilitate access, availability and legibility, which encourage collaboration among health care professionals (1). Arguably more important, EMRs decrease medical errors by providing 'complete' patient information and history (1).

Despite the purported benefits, evidence regarding the effect of EMRs on patient and health care team outcomes is limited (2). Furthermore, specific individual experiences concerning EMRs are poorly documented, contributing to challenges in determining their efficacy (2). Assessing their strengths and weaknesses is important because EMRs are associated with substantial financial implications, and ineffective EMRs would represent a waste of the vast resources required to implement and maintain EMR systems (3). More importantly, ineffective records can seriously undermine patient safety and lead to a significant number of medical errors.
Most studies suggest that EMRs provide accurate and complete patient records $(1,4,5)$. However, we had noticed both quantitative and qualitative inaccuracies (eg, wrong medications) and incompleteness (eg, incomplete documentation of medications and allergies) in EMRs obtained from referring physicians, and decided to evaluate the accuracy and completeness of EMRs. Tse and You (6) have previously noted discrepancies; however, this was a pilot study with several methodological limitations. The purpose of our study was to assess the feasibility of auditing EMRs in plastic surgery for a future large-scale research study. The secondary objective was to ascertain the accuracy and completeness of EMRs accompanying referral requests by physicians for plastic surgery consultation between July 15 and December 15, 2013.

\section{METHODS}

The present analysis was a prospective cohort study. The Hamilton Integrated Research Ethics Board (Hamilton, Ontario) provided approval (Project Number: 13-526-C).

${ }^{1}$ Faculty of Medicine, University of Ottawa, Ottawa; ${ }^{2}$ Division of Plastic Surgery; ${ }^{3}$ Surgical Outcomes Research Center (SOURCE), Department of Surgery; ${ }^{4}$ Department of Clinical Epidemiology and Biostatistics, McMaster University, Hamilton, Ontario

Correspondence: Dr Achilleas Thoma, Department of Surgery, Division of Plastic Surgery, McMaster University, 101-206 James Street South, Hamilton, Ontario L8P 3A9. Telephone 905-523-0019 fax 905-523-0229, e-mail athoma@mcmaster.ca 
TABLE 1

\section{Patient demographics}

\begin{tabular}{lc}
\hline Patients, $\mathrm{n}$ & 30 \\
Referring physician & \\
Family physician & $25(83.3)$ \\
Specialist & $5(16.7)$ \\
Time from when EMR was received to when patient was seen, days \\
Mean \pm SD & $95.6 \pm 57.7$ \\
Median (minimum, maximum) & $106.5(7,216)$
\end{tabular}

Data presented as $n$ (\%) unless otherwise indicated. EMR Electronic medical record

\section{Data collection}

The setting for the present study was the practice of the senior author (AT), a plastic surgeon practicing in Hamilton, Ontario. This is a diverse plastic surgery practice that includes both reconstructive and cosmetic adult patients. His patients are referred from Hamilton and the surrounding area, specifically the Hamilton Niagara Haldimand Brant Local Health Integration Network. In the course of the patient consultation, a complete up-to-date medical record was documented to include patient demographics, reason for referral, history of presenting illness, previous surgical/medical history, current medications and medical allergies. A standardized form designed a priori that included these information fields was used to collect data by the senior author and, subsequently, to compare against EMRs obtained from referring physicians. To minimize recording patient information that had changed between the time patients were referred and seen at the plastic surgery clinic, the senior author asked each patient whether any information had changed during the wait period. This was performed for each information field.

\section{Inclusion and exclusion criteria}

In the present study, all patients were included provided they had accompanying EMRs from their referring physicians.

\section{Data abstraction}

Using a data abstraction form in Excel (Microsoft Corporation, USA), data were extracted by two reviewers $(\mathrm{CH}, \mathrm{MK})$ independently. The reviewers crosschecked the EMRs of 30 patients referred between July 15 and December 15, 2013 with the senior author's medical records to assess their accuracy and completeness. The definition used for an inaccurate record was one that had a qualitative or quantitative error, while the definition used for an incomplete record was one that omitted information necessary to gain a full perspective on the patient and/or the nature of patient's disease. These definitions did not apply to patient information that had changed between the time patients were referred and seen at the plastic surgery clinic, as determined by the senior author at the time of patient consultation. In addition, the definition of an inaccurate record did not include spelling errors.

Data extracted/reviewed included patient demographics (ie, health insurance number, age, date of birth, sex), reason for referral, history of presenting illness, previous surgical/medical history, current medications and medical allergies. Each patient was given a unique identifier number to ensure confidentiality and privacy of the patients. Discrepancies in data abstraction were settled through consensus and, in the case of persistent discrepancies, discussion with a third reviewer (AT). In addition, the senior author reviewed every EMR information field that was deemed to be inaccurate or incomplete by the reviewers. To ensure the uniformity of data abstraction, each reviewer completed a calibration exercise before beginning data abstraction. The kappa statistic was calculated to determine concordance between the reviewers.

\section{Sample size calculation and statistical analyses}

Because the present analysis was a feasibility study, sample size was not calculated. Based on the data collected and extracted, descriptive
TABLE 2

Accuracy and completeness of electronic medical records

\begin{tabular}{lllllc}
\hline & \multicolumn{2}{c}{ Accurate } & & \multicolumn{2}{c}{ Complete } \\
\cline { 2 - 3 } \cline { 5 - 6 } & \multicolumn{1}{c}{ Yes } & No & & Yes & No \\
\hline Age/date of birth & $30(100)$ & $0(0)$ & & $30(100)$ & $0(0)$ \\
Reason for referral & $30(100)$ & $0(0)$ & & $30(100)$ & $0(0)$ \\
History of & $30(100)$ & $0(0)$ & & $8(26.7)$ & $22(73.3)$ \\
$\quad$ presenting illness & & & & \\
Medical history & $29(96.7)$ & $1(3.3)$ & & $18(60.0)$ & $12(40.0)$ \\
Surgical history & $30(100)$ & $0(0)$ & & $11(36.7)$ & $19(63.3)$ \\
Current medications & $28(93.3)$ & $2(6.7)$ & & $24(80.0)$ & $6(20.0)$ \\
Medical allergies & $29(96.7)$ & $1(3.3)$ & & $23(76.7)$ & $7(23.3)$ \\
Health insurance & $30(100)$ & $0(0)$ & & $30(100)$ & $0(0)$ \\
$\quad$ number & & & & & \\
Sex & $30(100)$ & $0(0)$ & & $30(100)$ & $0(0)$ \\
\hline
\end{tabular}

Data presented as $n$ (\%)

analysis was performed to calculate the frequency of inaccuracies and incompleteness for each EMR information field. Information fields were compared to assess whether the frequency of inaccuracies and incompleteness varied.

\section{RESULTS}

In the present study, the level of concordance for the calibration exercise was determined to be $\geq 90 \%$. Table 1 summarizes the demographics of the 30 patients, along with the specialties of referring physicians (ie, family physicians versus specialists) and time from when EMR was received to when the patient was seen. Notably, the majority of patients were referred by family physicians and the mean wait period was 95.6 days. Table 2 is a descriptive analysis demonstrating the frequency of inaccuracies and incompleteness for each EMR information field. The data revealed that all EMRs had accurate and complete age/ date of birth, reason for referral, health insurance number and sex. However, inaccuracies and/or incompleteness were found in all other information fields. Inaccuracies were found in the following information fields: current medications $(n=2)$, medical history $(n=1)$ and medical allergies $(n=1)$ (Figure 1). Figure 2 presents a pie chart of incompleteness in EMRs, with each segment representing an information field. The most common field of incompleteness was history of presenting illness $(n=22)$, followed by surgical history $(n=19)$ and medical history $(n=12)$.

\section{DISCUSSION}

The present analysis was the first comprehensive feasibility study to evaluate the frequency of inaccuracies and incompleteness in EMRs obtained from referring physicians over a five-month period. Despite the commonly held belief that EMRs decrease medical errors by providing 'complete' patient information and history, our results indicate that inaccuracies and incompleteness are a frequently occurring problem. Of the 270 EMR information fields that we reviewed, four $(1.48 \%)$ were inaccurate and $66(24.4 \%)$ were incomplete.

The critical errors identified in the present study are important because they potentially affect patient safety. For instance, only 22 of 30 EMRs analyzed had accurate and complete current medications and medical allergies, meaning that in eight cases, wrong medications could have been administered with possible serious consequences. Furthermore, 22 EMRs had incomplete history of presenting illness, which delayed the assessment and treatment of these patients. This suggests that there is a considerable discordance between an ideal setting in which EMRs reduce medical errors and enhance quality of care, and a practical setting in which EMRs are often associated with inaccuracies and incompleteness.

Interestingly, there was a considerable variation among EMR information fields, with several having no inaccuracies or incompleteness, and others, such as history of presenting illness, having frequencies of 


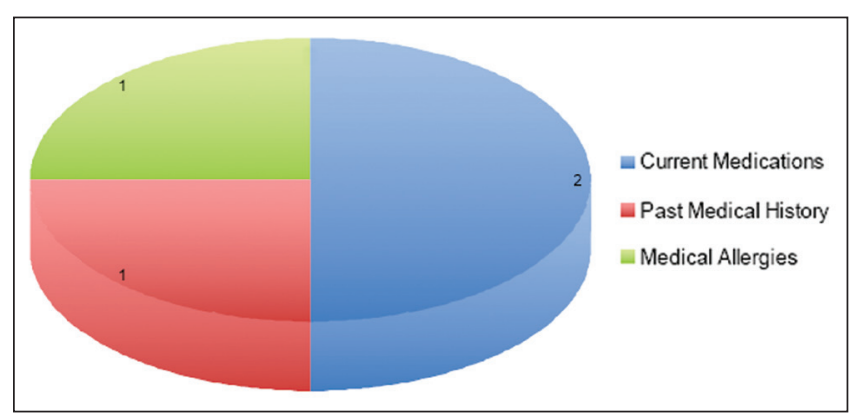

Figure 1) Inaccuracies in electronic medical records. Each segment represents a corresponding information field

incompleteness as high as 22 per 30 medical records. This may be explained, in part, by the fact that in many cases referring physicians used EMRs to simply denote their reason for referral rather than documenting complete patient information and history. EMRs are highly user dependent, and reported clinical benefits of EMRs are likely highly dependent on meaningful use and longer EMR experience by referring physicians (5).

\section{Comparison with previous publication}

In 2001, Tse and You (6) evaluated the frequency of inaccuracies and incompleteness in EMRs and found that 39\% and 51\% of information on allergies and medications, respectively, were inaccurate. Furthermore, they found a relatively high percentage (36\%) of incomplete information. The frequency of inaccuracies and incompleteness found in the present study was considerably lower compared with the frequency reported by Tse and You (6). The difference in our findings was likely the result of variance in study design, with the criteria applied in our study being more thorough and stringent.

\section{Strengths and weaknesses}

There were several limitations to the present study. First, because it relied on patient interviews to obtain data, recall bias was a possibility. In addition, because the senior author conducted patient interviews, he may have been prone to asking questions and documenting patient history more thoroughly. He likely collected more accurate and more complete data. However, in contrast, the senior author's thorough interviews and more accurate/complete data were advantageous because they were considered to be the gold standard in our study. The subjective nature of 'completeness' is another important limitation: what a referring physician considers to be 'complete' may not be 'complete' for a plastic surgeon in a tertiary care centre. Finally, the study was underpowered to draw definitive conclusions. To increase precision and internal validity, we needed to review at least 292 EMRs to achieve $95 \%$ CIs of $\pm 2.5 \%$ for accuracy and completeness of $\geq 95 \%$.

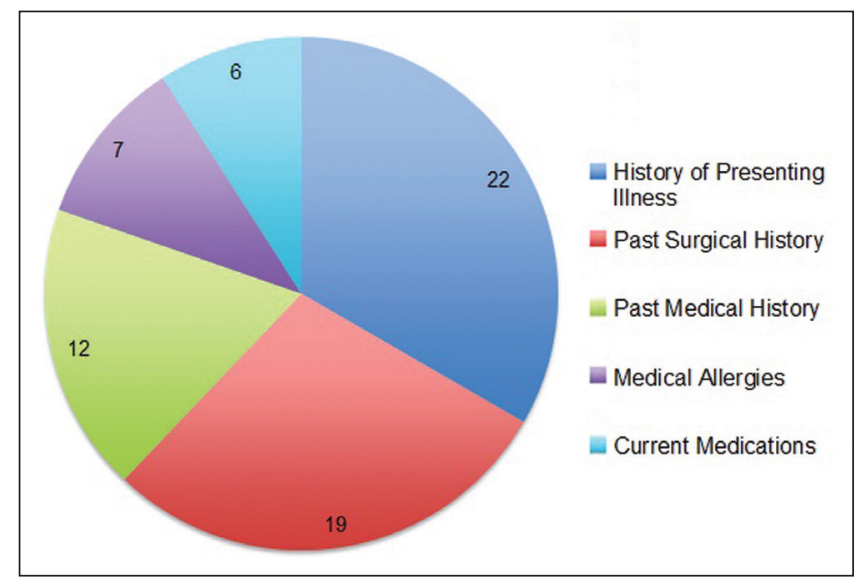

Figure 2) Incompleteness in electronic medical records. Each segment represents an information field

\section{CONCLUSION}

Our findings suggest that, despite the purported benefits of EMRs, they often contain inaccurate and incomplete patient information and history. Evidence from the present study suggests that a future large-scale study auditing EMRs to assess their efficacy is feasible.

ACKNOWLEDGEMENTS: The authors thank Angela Barbas and Dyda Dao for their assistance in filing standardized forms for data collection and statistical analyses, respectively.

DISCLOSURE: The authors have no financial disclosures or conflicts of interest to declare.

\section{REFERENCES}

1. Cimino JJ. Improving the electronic health record - are clinicians getting what they wished for? JAMA 2013;309:991-2.

2. Terry AL, Thorpe CF, Giles G, et al. Implementing electronic health records: Key factors in primary care. Can Fam Physician 2008;54:730-6.

3. Fleming NS, Becker ER, Culler SD, et al. The impact of electronic health records on workflow and financial measures in primary care practices. Health Serv Res 2013 (Epub ahead of print).

4. Bates DW, Teich JM, Lee J, et al. The impact of computerized physician order entry on medication error prevention. J Am Med Inform Assoc 1999;6:313-21.

5. King J, Patel V, Jamoom EW, et al. Clinical benefits of electronic health record use: National findings. Health Serv Res 2013 (Epub ahead of print).

6. Tse J, You W. How accurate is the electronic health record? - a pilot study evaluating information accuracy in a primary care setting. Stud Health Technol Inform 2011;168:158-64. 